\title{
BMJ
}

\section{Nature of socioeconomic inequalities in neonatal mortality: population based study}

\author{
Lucy K Smith, research fellow, ${ }^{1}$ Bradley N Manktelow, statistician, ${ }^{1}$ Elizabeth S Draper, professor of perinatal \\ and paediatric epidemiology, ${ }^{1}$ Anna Springett, data analyst, ${ }^{2}$ David J Field, professor of neonatal medicine ${ }^{1}$
}

${ }^{1}$ Department of Health Sciences, University of Leicester, Leicester LE1 6TP, UK

${ }^{2}$ Centre for Maternal and Child Enquiries, London NW1 5SD, UK Correspondence to: L K Smith Iks1@leicester.ac.uk

Cite this as: BMJ 2010;341:c6654 doi:10.1136/bmj.c6654

\section{ABSTRACT}

Objective To investigate time trends in socioeconomic inequalities in cause specific neonatal mortality in order to assess changing patterns in mortality due to different causes, particularly prematurity, and identify key areas of focus for future intervention strategies.

Design Retrospective cohort study.

Setting England.

Participants All neonatal deaths in singleton infants born between 1 January 1997 and 31 December 2007.

Main outcome measure Cause specific neonatal mortality per 10000 births by deprivation tenth (deprivation measured with UK index of multiple deprivation 2004 at super output area level).

Results 18524 neonatal deaths occurred in singleton infants born in the 11 year study period. Neonatal mortality fell between 1997-9 and 2006-7 (from 31.4 to 25.1 per 10000 live births). The relative deprivation gap (ratio of mortality in the most deprived tenth compared with the least deprived tenth) increased from 2.08 in 1997-9 to 2.68 in 2003-5, before a fall to 2.35 in 2006-7. The most common causes of death were immaturity and congenital anomalies. Mortality due to immaturity before 24 weeks' gestation did not decrease over time and showed the widest relative deprivation gap (2.98 in 1997 9; 4.14 in 2003-5; 3.16 in 2006-7). Mortality rates for all other causes fell over time. For congenital anomalies, immaturity, and accidents and other specific causes, the relative deprivation gap widened between $1997-9$ and 2003-5, before a slight fall in 2006-7. For intrapartum events and sudden infant deaths (only $13.5 \%$ of deaths) the relative deprivation gap narrowed slightly.

Conclusions Almost $80 \%$ of the relative deprivation gap in all cause mortality was explained by premature birth and congenital anomalies. To reduce socioeconomic inequalities in mortality, a change in focus is needed to concentrate on these two influential causes of death. Understanding the link between deprivation and preterm birth should be a major research priority to identify interventions to reduce preterm birth.

\section{INTRODUCTION}

Socioeconomic inequalities in infant mortality exist in many developed countries despite improvements in overall mortality. ${ }^{12}$ Countries have varying strategies to reduce these inequalities. ${ }^{1}$ The UK government has made major attempts to tackle socioeconomic inequalities in infant mortality by setting a public service agreement target in 2003 to reduce the relative deprivation gap in England and Wales by $10 \%$ by 2010. However, recent evidence indicates that this target is unlikely to be achieved and the relative deprivation gap may be widening. ${ }^{4}$ Public health interventions targeting major potentially modifiable risk factors for mortality are essential. Design of such interventions requires an understanding of current trends in cause specific mortality, as specific causes of death are likely to have different effects on the widening relative deprivation gap in all cause mortality. Recent UK evidence on inequalities in cause specific mortality is contradictory and predominantly cross sectional, preventing the investigation of trends over time. ${ }^{56}$

Premature birth is the major component of neonatal and infant mortality, accounting for two thirds of neonatal deaths in England. ${ }^{7}$ Most of these deaths result from very preterm birth (<33 completed weeks' gestation), ${ }^{7}$ and rates of delivery at less than 33 weeks' gestation are rising in the United Kingdom and internationally. ${ }^{89}$ Research has also shown wide socioeconomic inequalities in the rates of premature birth. ${ }^{810}$ The combined effect of these trends could have a major effect on the deprivation gap in all cause mortality.

We did a cause specific analysis of time trends in socioeconomic inequalities in neonatal mortality in England over an 11 year period to understand the effect of specific causes of death on the widening relative deprivation gap in all cause mortality.

\section{METHODS}

Data on all neonatal deaths (death before 28 days of life) of singleton infants born to mothers resident in England between 1 January 1997 and 31 December 2007 came from the Centre for Maternal Child Enquiries (CMACE; www.cmace.org.uk), which has collected neonatal mortality data as part of its national perinatal mortality surveillance work funded by the National Patient Safety Agency. Data included cause of death, gestational age, and super output area (geographical populations of approximately 1500 residents) of mother's residence. Since 1 January 2004, 
Table 1| Number of live births and neonatal deaths (percentage of neonatal deaths) by deprivation tenth and cause of death 1997-2007

\begin{tabular}{|c|c|c|c|c|c|c|c|c|c|c|c|}
\hline \multirow[b]{2}{*}{ Cause of death } & \multicolumn{10}{|c|}{ Deprivation tenth (1=least deprived; $10=$ most deprived) } & \multirow[b]{2}{*}{ All } \\
\hline & 1 & 2 & 3 & 4 & 5 & 6 & 7 & 8 & 9 & 10 & \\
\hline Live births & 644569 & 641660 & 642828 & 642752 & 642206 & 640154 & 638287 & 637269 & 637291 & 642675 & 6409691 \\
\hline \multicolumn{12}{|l|}{ Neonatal deaths: } \\
\hline All causes & $1159(6.3)$ & $1298(7.0)$ & $1417(7.6)$ & $1623(8.8)$ & $1622(8.8)$ & $1818(9.8)$ & $2038(11.0)$ & $2213(11.9)$ & $2536(13.7)$ & $2800(15.1)$ & $18524(100)$ \\
\hline Congenital anomalies & 288 & 315 & 333 & 371 & 357 & 392 & 464 & 549 & 657 & 738 & $4464(24.1)$ \\
\hline Intrapartum events & 170 & 166 & 188 & 196 & 188 & 225 & 211 & 216 & 205 & 194 & $1959(10.6)$ \\
\hline Immaturity <24 weeks' gestation & 189 & 211 & 231 & 290 & 301 & 334 & 441 & 430 & 532 & 643 & $3602(19.4)$ \\
\hline Immaturity 24-27 weeks' gestation & 233 & 251 & 289 & 305 & 332 & 392 & 394 & 421 & 475 & 511 & $3603(19.5)$ \\
\hline Immaturity $28-36$ weeks' gestation & 57 & 77 & 62 & 96 & 78 & 101 & 91 & 109 & 109 & 136 & $916(4.9)$ \\
\hline Immaturity (gestation unknown) & 2 & 6 & 5 & 10 & 11 & 9 & 12 & 16 & 22 & 24 & $117(0.6)$ \\
\hline Infection & 93 & 126 & 118 & 165 & 152 & 180 & 172 & 203 & 229 & 239 & $1677(9.1)$ \\
\hline Accident and other specific causes & 75 & 95 & 127 & 114 & 116 & 105 & 161 & 156 & 168 & 178 & $1295(7.0)$ \\
\hline Sudden infant death & 32 & 38 & 33 & 46 & 52 & 56 & 54 & 79 & 81 & 72 & $543(2.9)$ \\
\hline Unclassified & 20 & 13 & 31 & 30 & 35 & 24 & 38 & 34 & 58 & 65 & 348 (1.9) \\
\hline
\end{tabular}

CMACE has no longer collected information on postneonatal mortality (death between 28 days and 1 year), preventing analyses of infant mortality for the whole 11 year period. However, we did sensitivity analyses using information on infant mortality in 1997-2003 to assess the differences in patterns between neonatal and infant mortality. Denominator data on all live singleton births by year of birth and super output area came from the UK Office for National Statistics (www.statis tics.gov.uk). We focused on singleton births because differential access to fertility treatment may lead to a higher incidence of multiple births in less deprived areas and the neonatal mortality of multiple births is higher than that of singletons.

A local CMACE coordinator initially classified neonatal deaths by using the extended Wigglesworth hierarchical classification system. ${ }^{11}$ A CMACE regional manager then checked them with reference to postmortem and coroner's reports where available. Finally, CMACE carried out central cross validation checks to ensure consistency. Here, we expanded the Wigglesworth classification for deaths due to immaturity, on the basis of gestational age at birth $(<24$ weeks, 24-27 weeks, and 28-36 weeks). We grouped accidental deaths with "other specific conditions."

The government targets in England and Wales measure the relative deprivation gap by using a classification of socioeconomic group based on father's occupation. $^{3}$ This excludes infants whose parents have never worked and those who are solely registered by the mother, and these infants have a greatly increased risk of death. We measured socioeconomic inequalities by using an area level measure of deprivation, the index of multiple deprivation for 2004 at the super output area level, to enable inclusion of all infants. ${ }^{12}$ This measure of multiple deprivation is made up of seven domain indices at the super output area level, which relate to income deprivation, employment deprivation, health deprivation and disability, education, skills and training deprivation, barriers to housing and services, and living environment deprivation and crime. Super output areas are the smallest areas for which these deprivation data are available; although some degree of heterogeneity will exist within them, the small size of the areas (only 1500 residents) limits this. We ranked all super output areas in England by deprivation score and divided them into 10 groups with approximately equal populations of births: 1 (least deprived) to 10 (most deprived). If neonatal mortality was the same for all deprivation groups, a similar number of neonatal deaths would be expected in each tenth.

We calculated neonatal mortality rates for each cause of death by deprivation tenth and time period (1997-9, 2000-2, 2003-5, and 2006-7). We used Poisson regression models to assess trends in mortality by deprivation tenth over time, ${ }^{13}$ fitting separate models for all cause mortality and each specific cause of death.

UK targets for reducing socioeconomic inequalities in infant mortality are based on the relative deprivation gap, to avoid the influence of the underlying prevalence. Here, to compare our findings with the national targets, we measured the relative deprivation gap by fitting a linear trend between deprivation tenth and mortality and calculating the mortality rate ratio between the most deprived and least deprived tenths, which is similar in approach to the relative index of inequality. ${ }^{14} \mathrm{We}$ assessed significant change in the relative deprivation gap over time by fitting a separate deprivation effect for each time period. We assessed reductions in neonatal mortality over time between 1997-9 and 2006-7 by relative change (percentage reduction in mortality rate by deprivation tenth). However, investigating both the relative and absolute deprivation gap can aid a better understanding of time trends in socioeconomic inequalities. Adjusting for the underlying prevalence can ignore important changes in the absolute deprivation gap. Here, we calculated absolute change in neonatal mortality over time by deprivation tenth (difference in neonatal mortality per 10000 births by deprivation tenth) to assess improvements in mortality. We used the delta method to calculate confidence limits. ${ }^{15} \mathrm{We}$ estimated excess mortality associated with deprivation as a percentage by applying the neonatal mortality rate in the 
Table 2| Observed neonatal mortality rate per 10000 live births by deprivation tenth and year of birth, and estimated reduction in mortality per 10000 births between 1997-9 and 2006-7 (based on Poisson regression model)

\begin{tabular}{|c|c|c|c|c|c|c|}
\hline \multirow[b]{2}{*}{ Cause of death } & \multicolumn{4}{|c|}{ Mortality per 10000 live births } & \multicolumn{2}{|c|}{ Reduction in mortality from $1997-9$ to $2006-7$} \\
\hline & 1997-9 & $2000-2$ & 2003-5 & 2006-7 & $\begin{array}{l}\text { Relative change (\%) } \\
\qquad(95 \% \mathrm{Cl})\end{array}$ & $\begin{array}{l}\text { Absolute change per } \\
10000 \text { births }(95 \% \mathrm{Cl})\end{array}$ \\
\hline \multicolumn{7}{|l|}{ All causes: } \\
\hline Least deprived tenth & 20.8 & 18.3 & 16.9 & 14.9 & 26.1 (18.9 to 32.7$)$ & 5.55 (3.89 to 7.21$)$ \\
\hline Most deprived tenth & 46.4 & 46.6 & 43.5 & 35.9 & 16.7 (10.4 to 22.6$)$ & 7.41 (4.49 to 10.32$)$ \\
\hline \multicolumn{7}{|l|}{ Congenital anomaly: } \\
\hline Least deprived tenth & 5.7 & 3.9 & 4.0 & 4.1 & 31.3 (16.9 to 43.3$)$ & $1.60(0.81$ to 2.39$)$ \\
\hline Most deprived tenth & 12.4 & 12.4 & 10.5 & 10.5 & $9.5(-4.6$ to 21.7$)$ & $1.05(-0.45$ to 2.56$)$ \\
\hline \multicolumn{7}{|l|}{ Intrapartum events: } \\
\hline Least deprived tenth & 2.4 & 2.7 & 3.1 & 2.2 & $15.6(-9.4$ to 34.9$)$ & $0.45(-0.23$ to 1.13$)$ \\
\hline Most deprived tenth & 3.6 & 2.5 & 3.2 & 2.6 & 29.4 (9.9 to 44.6$)$ & 1.17 (0.37 to 1.96$)$ \\
\hline \multicolumn{7}{|c|}{ Immaturity <24 weeks' gestation: } \\
\hline Least deprived tenth & 2.6 & 3.5 & 2.9 & 2.7 & $1.9(-22.4$ to 21.4$)$ & $0.06(-0.58$ to 0.69$)$ \\
\hline Most deprived tenth & 8.6 & 10.7 & 11.2 & 9.3 & $-4.0(-22.0$ to 11.3$)$ & $-0.35(-1.76$ to 1.07$)$ \\
\hline \multicolumn{7}{|c|}{ Immaturity 24-27 weeks' gestation: } \\
\hline Least deprived tenth & 4.9 & 3.4 & 3.1 & 2.7 & $44.7(31.4$ to 55.4$)$ & $2.19(1.44$ to 2.94$)$ \\
\hline Most deprived tenth & 9.9 & 8.0 & 7.4 & 6.1 & 35.1 (22.9 to 45.4$)$ & $3.24(2.00$ to 4.48$)$ \\
\hline \multicolumn{7}{|c|}{ Immaturity 28-36 weeks' gestation: } \\
\hline Least deprived tenth & 1.0 & 1.1 & 0.8 & 0.5 & 56.8 (32.3 to 72.4$)$ & $0.81(0.42$ to 1.21$)$ \\
\hline Most deprived tenth & 3.0 & 1.8 & 2.4 & 0.9 & 60.4 (42.3 to 72.8$)$ & $1.63(1.03$ to 2.23$)$ \\
\hline \multicolumn{7}{|l|}{ Infection: } \\
\hline Least deprived tenth & 1.8 & 1.4 & 1.3 & 1.2 & $24.6(-1.9$ to 44.3$)$ & $0.49(-0.02$ to 0.99$)$ \\
\hline Most deprived tenth & 3.7 & 4.2 & 3.7 & 3.1 & $-5.2(-33.0$ to 16.8$)$ & $-0.20(-1.11$ to 0.72$)$ \\
\hline \multicolumn{7}{|c|}{ Accidents and other specific causes: } \\
\hline Least deprived tenth & 1.8 & 0.9 & 1.0 & 0.8 & $10.7(-24.5$ to 35.9$)$ & $0.17(-0.32$ to 0.66$)$ \\
\hline Most deprived tenth & 2.8 & 3.2 & 2.6 & 2.4 & $7.2(-23.0$ to 29.9$)$ & $0.19(-0.53$ to 0.91$)$ \\
\hline \multicolumn{7}{|l|}{ Sudden infant death: } \\
\hline Least deprived tenth & 0.3 & 0.9 & 0.3 & 0.4 & $6.8(-69.0$ to 48.6$)$ & $0.03(-0.21$ to 0.27$)$ \\
\hline Most deprived tenth & 1.4 & 1.3 & 1.1 & 0.5 & $40.3(6.9$ to 61.7$)$ & $0.62(0.11$ to 1.14$)$ \\
\hline
\end{tabular}

least deprived tenth to the total population and dividing that by the total number of deaths observed. We calculated the proportion of the deprivation gap in all cause neonatal mortality explained by each cause for each time period. For each specific cause, we estimated the neonatal mortality rate in the least deprived tenth and the most deprived tenth for each time period by using the regression models. We calculated the absolute difference in these two rates and expressed it as a proportion of the absolute difference in rates for all causes combined.

\section{RESULTS}

All cause neonatal mortality

CMACE received notification of 18524 neonatal deaths of singleton infants between 1997 and 2007. The number of deaths increased with increasing deprivation (table 1). All cause neonatal mortality fell over time from 31.4 per 10000 live births in $1997-9$ to 25.1 per 10000 live births in 2006-7. In absolute terms, rates decreased more between 1997-9 and 2006-7 in the most deprived tenth ( 7.4 fewer deaths per 10000 births in most deprived tenth; 5.6 fewer deaths per 10000 births in least deprived tenth). However, the relative reduction in mortality over time was smaller in the most deprived tenth $(17 \%)$ compared with the least deprived tenth (26\%) (table 2). In 1997-9 infants were at twice the risk of neonatal death in the most deprived tenth compared with the least deprived tenth (mortality rate ratio $2.08,95 \%$ confidence interval 1.92 to 2.27 ) (table 3). This gap widened significantly over time to a peak of 2.68 in 2003-5 and narrowed slightly to 2.35 in 2006-7. Consequently, the percentage of excess deaths associated with deprivation increased over the time period from $32.3 \%$ in $1997-9$ to $51.0 \%$ in $2003-5$ and then decreased to $37.5 \%$ in $2006-7$ (table 4 ).

\section{Cause specific neonatal mortality}

Deaths due to immaturity were the most common (44.5\%), followed by those due to congenital anomalies (24.1\%), intrapartum events $(10.6 \%)$, infection $(9.1 \%)$, accidents and other specific causes $(7.0 \%)$, and sudden infant deaths $(2.9 \%)$ (table 1). The number of deaths increased with increasing deprivation for each cause, although the increase varied in magnitude. With the exception of deaths due to immaturity at less than 24 weeks' gestation, neonatal mortality fell over time for all causes; the greatest falls were for immaturity at 24-27 weeks' and 28-36 weeks' gestation.

For five of the eight causes of death (congenital anomalies, immaturity at $<24$ and $24-27$ weeks, infection, and accidents and other specific causes), a larger 
Table $3 \mid$ Neonatal mortality rate ratio comparing most deprived tenth with least deprived tenth by time period and cause of death (based on Poisson regression model)

\begin{tabular}{|c|c|c|c|c|c|}
\hline Cause of death & $1997-9$ & $2000-2$ & 2003-5 & $2006-7$ & $P$ value* \\
\hline All causes & 2.08 (1.92 to 2.27$)$ & $2.53(2.32$ to 2.77$)$ & $2.68(2.45$ to 2.93$)$ & $2.35(2.10$ to 2.63$)$ & 0.0004 \\
\hline Congenital anomaly & $2.16(1.82$ to 2.56$)$ & $2.92(2.43$ to 3.51$)$ & $3.06(2.54$ to 3.70$)$ & $2.85(2.27$ to 3.58$)$ & 0.0264 \\
\hline Intrapartum events & 1.37 (1.07 to 1.76$)$ & $1.15(0.87$ to 1.53$)$ & $1.21(0.94$ to 1.56$)$ & $1.15(0.82$ to 1.61$)$ & 0.7817 \\
\hline Immaturity <24 weeks' gestation & $2.98(2.42$ to 3.67$)$ & $3.28(2.66$ to 4.05$)$ & $4.14(3.40$ to 5.06$)$ & $3.16(2.47$ to 4.04$)$ & 0.1217 \\
\hline Immaturity 24-27 weeks' gestation & 1.88 (1.57 to 2.25$)$ & $2.49(2.03$ to 3.06$)$ & 2.38 (1.94 to 2.92$)$ & 2.21 (1.68 to 2.91$)$ & 0.1779 \\
\hline Immaturity 28-36 weeks' gestation & 1.88 (1.35 to 2.62$)$ & 1.74 (1.16 to 2.59$)$ & $3.22(2.10$ to 4.95$)$ & $1.72(0.93$ to 3.18$)$ & 0.1393 \\
\hline Infection & $1.92(1.45$ to 2.54$)$ & $2.66(2.00$ to 3.53$)$ & 2.17 (1.57 to 3.00$)$ & 2.68 (1.88 to 3.83$)$ & 0.3333 \\
\hline Accidents and other specific causes & $1.70(1.23$ to 2.35$)$ & 2.29 (1.65 to 3.18$)$ & 2.71 (1.92 to 3.83$)$ & 1.77 (1.18 to 2.65$)$ & 0.1987 \\
\hline Sudden infant death & 3.62 (2.15 to 6.07$)$ & $2.47(1.49$ to 4.07$)$ & 2.08 (1.26 to 3.43$)$ & 2.32 (1.14 to 4.73$)$ & 0.4806 \\
\hline
\end{tabular}

relative fall in mortality occurred over time in the least deprived tenth compared with the most deprived tenth (table 2), although this was statistically significant only for congenital anomalies. For these causes and immaturity at 28-36 weeks, the trend in the deprivation gap over time was similar to that for all cause neonatal mortality, with an initial wide deprivation gap in 1997-9 (neonatal mortality rate ratio range $1.70-2.98$ ), which increased up to 2003-5 (range 2.17-4.14) followed by a slight narrowing in 2006-7 (range 1.72-3.16). The widest deprivation gap was for immaturity at less than 24 weeks' gestation; those from the most deprived tenth had a threefold risk of death in 1997-9 compared with the least deprived tenth, which increased to more than a fourfold risk in 2003-5 and slightly reduced to a threefold risk in 2006-7. As the mortality rate for these causes did not fall over the time period, this widening of the deprivation gap meant that they represented a larger proportion of all deaths in 2006-7 (21.7\%) than in 1997-9 (16.9\%).

Intrapartum deaths and sudden infant deaths showed a greater fall in mortality in the most deprived tenth than in the least deprived tenth, leading to a nonsignificant narrowing of the deprivation gap for these causes. However, these deaths comprised only $13.5 \%$ of deaths and their effect on all cause mortality was small. Deaths due to intrapartum events showed the narrowest deprivation gap in mortality rates (range 1.15-1.37). Consequently, the reduction in deaths if the rates seen in the least deprived tenth were applied across all tenths was small (range 6.8-14.8\%). In contrast, the deprivation gap for sudden infant deaths was the widest seen for any specific cause in 1997-9 (mortality rate ratio 3.62). This fell non-significantly over time to 2.32 in 2006-7. Sudden infant deaths would have been reduced by more than half in 1997-9 if mortality rates for the least deprived tenth were applied to the whole population, compared with a reduction of just over a third in 2006-7.

The figure shows the percentage of the deprivation gap in all cause neonatal mortality explained by each specific cause. Deaths due to immaturity (babies $<24$ weeks', 24-27 weeks', and 28-36 weeks' gestation) and congenital anomalies explain most of the deprivation gap in all cause mortality, which increased from
$77 \%$ in $1997-9$ to a peak of $82 \%$ in $2003-5$ and then fell to $79 \%$ in $2006-7$. This results from a combination of a widening deprivation gap in mortality for these causes, higher mortality, and a lack of decline in mortality due to immaturity at less than 24 weeks. The remaining causes (sudden infant death, intrapartum events, infection, and accidents and other causes) account for only $20 \%$ of the deprivation gap. This is partially related to lower mortality but also to the narrow deprivation gap in mortality for intrapartum deaths. The percentage of the gap explained by sudden infant deaths fell over time from $5 \%$ in $1997-9$ to $2.5 \%$ in $2006-7$.

\section{Sensitivity analyses}

Sensitivity analyses of infant mortality (1997-2003) showed extremely similar trends in the deprivation gap over time for all cause mortality and each specific cause but with slightly wider deprivation gaps than for neonatal mortality. In 1997-9 sudden infant deaths explained $20 \%$ of the deprivation gap in all cause infant mortality, but this declined to $8 \%$ by 2003 . By contrast, immaturity and congenital anomalies accounted for $53 \%$ of the deprivation gap in 1997-9, which increased to $73 \%$ in 2003 , similar to the percentage seen for neonatal mortality. Hence, whereas the percentage of infant deaths due to each cause differed from neonatal deaths in 1997-9, over time the patterns are becoming more similar as immaturity and congenital anomalies play a greater role.

\section{DISCUSSION}

This study estimated time trends in the deprivation gap in neonatal mortality by cause of death, for which limited data have been published. Neonatal mortality rates were more than twice as high in the most deprived areas of England than in the least deprived areas, and the relative gap widened over time before a slight narrowing in recent years. Neonatal deaths would be 39\% lower if all areas had the same neonatal mortality rates as the least deprived areas. This widening relative deprivation gap in all cause neonatal mortality is particularly associated with an increase in the proportion of deaths associated with immaturity at less than 24 weeks ' gestation, for which the deprivation gap in mortality is widest, and differential falls in mortality by deprivation 
Table $4 \mid$ Excess neonatal mortality associated with deprivation by cause of death and time period. Values are percentages

\begin{tabular}{lcccc} 
Cause of death & $1997-9$ & $2000-2$ & $2003-5$ & $2006-7$ \\
All causes & 32.3 & 39.6 & 51.0 & 37.5 \\
\hline Congenital anomaly & 33.7 & 44.5 & 46.5 & 44.3 \\
\hline Intrapartum events & 14.8 & 6.9 & 9.3 & 6.8 \\
\hline Immaturity <24 weeks' gestation & 45.1 & 48.4 & 55.7 & 47.8 \\
\hline Immaturity 24-27 weeks' gestation & 28.3 & 39.0 & 37.7 & 35.2 \\
\hline Immaturity 28-36 weeks' gestation & 28.3 & 25.1 & 48.1 & 25.3 \\
\hline Infection & 29.1 & 41.3 & 34.3 & 42.2 \\
\hline Accidents and other specific causes & 24.2 & 35.9 & 42.3 & 26.3 \\
\hline Sudden infant death & 51.2 & 38.7 & 32.6 & 37.0
\end{tabular}

over time for congenital anomalies and immaturity at 24-27 weeks' and 28-36 weeks' gestation. Wide socioeconomic inequalities existed in deaths due to congenital anomalies and immaturity, as seen previously, and these causes accounted for more than three quarters of the deprivation gap in all cause mortality.

The relative deprivation gap in all cause neonatal mortality reported here is wider than that in the UK government's report on infant mortality, ${ }^{4}$ which compared routine and manual workers with the most advantaged occupational group (2005 rate ratio 1.94). However, the gap we have identified is similar to that found in a comparison of solely registered births and those whose father has never worked with the most advantaged group (2005 rate ratio 2.78). ${ }^{1}$ To properly understand the current situation and the effect of any intervention, these high risk infants must not be overlooked and should be included in analyses.

Although we have shown that the relative deprivation gap in neonatal mortality has generally widened over time, the absolute gap has reduced. The fall in neonatal mortality in the most deprived areas has been greater in absolute terms than in the least deprived areas. Consequently, although the UK government targets are unlikely to be achieved, efforts to improve neonatal mortality in the most deprived areas over the past decades have been successful to some extent.

Premature birth has a major impact on the deprivation gap, particularly for deaths among infants born at less than 24 weeks' gestation, who show the widest deprivation gap in neonatal mortality and, unlike for all other causes of death, no evidence of a decline in mortality over time. Tertiary interventions to reduce the burden of mortality and morbidity, such as advances in neonatal care, have led to great improvements in survival of premature infants born at 24-32 weeks' gestation. ${ }^{9}$ However, the extremely poor survival of those born at less than 24 weeks' gestation has changed little over time in the $\mathrm{UK},{ }^{16}$ and this is also true of population based data from elsewhere in the world. ${ }^{1718}$ We believe that further progress in neonatal care alone is unlikely to change this situation. As a result, the impact of extremely preterm infants on neonatal mortality is likely to increase, as their rates are increasing in the UK and internationally. ${ }^{89}$ Changes in practice as to whether babies are considered to be live births may partially explain this increase in incidence. Babies born dead before 24 weeks' gestation are not part of the mandatory registration scheme, and trends in the overall birth rate (alive and dead) at this gestation cannot be reliably estimated. We believe that mechanisms that lead to birth at this gestation are likely to be largely the same as those for babies born prematurely after 24 weeks. As a consequence, they are almost certainly part of the spectrum of increased risk of preterm birth that seems to be associated with deprivation. ${ }^{819}$ Our data from an English region suggest that, over time, a greater proportion of these babies are considered to be live births, increasing their contribution to all cause mortality. ${ }^{16}$ This is in stark contrast to the Netherlands, where policy is in place to attempt resuscitation of infants only if they are 25 weeks' gestation and over. ${ }^{20}$

Socioeconomic inequalities in deaths due to congenital anomalies are widening and represent more than a third of the deprivation gap in all cause mortality. Various potential underlying trends may contribute to the widening gap. These include whether the birth prevalence of certain lethal anomalies has decreased among the less deprived groups and similarly whether these mothers have differential access to or uptake of treatments available for the most severe anomalies. Maternal factors such as age and ethnicity also influence these factors. Variations exist in the UK on a regional basis, but whether they are due to underlying socioeconomic differences is not known. ${ }^{21}$ Increased risk of non-chromosomal congenital anomalies has been seen with increasing deprivation, ${ }^{2223}$ and recent declines in these anomalies may be more evident among the least deprived communities. ${ }^{21}$ Of equal importance are socioeconomic differences in women's attitudes to termination when a severe anomaly is confirmed. Further detailed research is needed to determine whether the observed differences represent variations in choice, differential access to screening and treatments, or changes in incidence.

Neonatal mortality rates for intrapartum events showed the least variation with deprivation, as previously seen, ${ }^{246}$ indicating that this aspect of midwifery

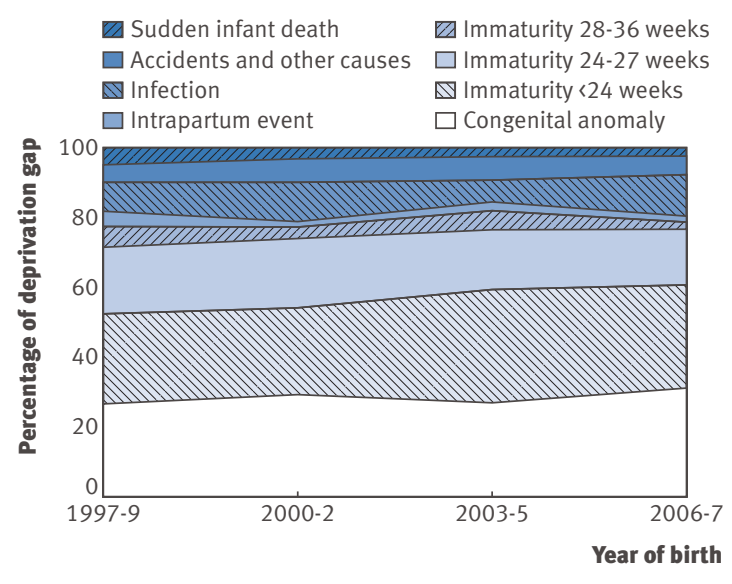

$\overline{\text { Percentage of deprivation gap in all cause mortality explained }}$ by each cause of death over time 


\section{WHAT IS ALREADY KNOWN ON THIS TOPIC}

Socioeconomic inequalities in infant and neonatal mortality exist in many developed countries, with a higher rate of early childhood death in families from deprived backgrounds

A government target was introduced to reduce the deprivation gap in infant mortality in England by $10 \%$ by 2010 , yet the gap has widened; an implementation strategy has been instigated to achieve this target

\section{WHAT THIS STUDY ADDS}

Almost $80 \%$ of the deprivation gap in neonatal mortality in England is the result of differences in deaths related to either immaturity or congenital anomalies

The current implementation strategy in England to reduce the relative deprivation gap does not adequately tackle these two main causes of death

Similar patterns probably exist in other developed countries, and these are best explored through cause specific analysis. and obstetric care is less influenced by deprivation. As such events are acute and generally not predictable before labour, this finding provides reassurance that care in labour is not related to deprivation. However, intrapartum deaths remain relatively common and mortality rates changed little over time, indicating that improvements to midwifery and obstetric care in England do not seem to have affected these deaths. The effect of public health campaigns to reduce the rate of sudden infant death is certainly reflected in our data, with declines in the deprivation gap, and the overall influence of this cause of death on neonatal mortality is now limited.

\section{Limitations}

Our work focused on neonatal mortality, as data on gestation specific post-neonatal deaths after 2003 were not available. However, patterns in infant mortality in 1997-2003 showed extremely similar patterns to those seen for neonatal mortality, with a slightly wider deprivation gap. The contribution of each cause to the overall deprivation gap is similar, and we believe that this similarity is likely to persist over time. Our main interest was in the effect of changing patterns of deaths relating to immaturity, and this is more likely to be seen in neonatal mortality. Our work lacks detailed data on individual risk behaviour and so may be open to problems of confounding. Epidemiological work using individual level data have shown wide differences in mortality-for example, mortality due to maternal smoking during pregnancy in infants from deprived areas in Scotland.$^{25}$ Despite this, provided the results are treated cautiously and trends are not extrapolated beyond the time period under study, our methods are relatively straightforward and provide a way for health service planners to monitor up to date trends in mortality.

\section{Implications}

Despite targets being set to improve inequalities in infant mortality by 2010 in the UK, the deprivation gap does not seem to be narrowing. Cause specific analyses provide more detailed information, highlighting the contribution of each causal group and the effect of interventions or changes in society over time. This study was based entirely on data for England, but we believe that cause specific analyses could provide much greater insight into socioeconomic inequalities in neonatal mortality on a global level and allow all countries to more fully understand their early childhood mortality rates and prioritise appropriate interventions.

In the absence of such an analysis, various identifiable actions have been recommended in England and Wales to reduce the deprivation gap in infant mortality by the target $10 \% 0^{4}$ (in order of magnitude of anticipated reduction): increasing breastfeeding rates and reducing obesity in the routine and manual work group; reducing smoking rates during pregnancy; and reducing rates of sudden infant death, overcrowded housing, and teenage conceptions in the routine and manual work group. Although these are all laudable aims, the work presented here shows that unless interventions target specifically the risk of very premature birth and potentially lethal congenital abnormalities the effect on the deprivation gap is likely to be minor. For example, even accounting for the higher rates of infant mortality due to sudden infant death compared with neonatal mortality, on the basis of our findings the contribution to the total mortality gap is simply too small to have an important effect. The situation is somewhat different for smoking, which evidence suggests is an important factor in the causes of preterm birth and as a consequence infant mortality. ${ }^{2021}$ The potential effect of reducing obesity and teenage pregnancy is less clear, in terms of existing evidence. ${ }^{622}$ However, smoking, obesity, and teenage pregnancy have all been the subject of longstanding public health campaigns with limited success, and the UK's suggested goals require major behavioural changes. Our lack of understanding about the everyday environmental influences on the risk of preterm birth and major congenital abnormalities seems to be a sig nificant impediment to the development of a rational strategy for diminishing the influence of deprivation on measures of early childhood mortality.

We have previously shown the importance of immaturity in the UK compared with other European countries, ${ }^{26}$ and the March of Dimes Foundation has highlighted the problem in global terms. ${ }^{9}$ Tackling the wide deprivation gap in deaths of babies born at less than 24 weeks' gestation is unlikely to be achievable through further progress in neonatal care, highlighting the need for a greater understanding of the mechanistic link between deprivation and prematurity. This should be a major research priority ${ }^{27}$ which would then allow a focus on primary preventive strategies to reduce the rate of prematurity itself. ${ }^{28}$ Our lack of understanding about the influence of health inequality in relation to major congenital anomalies deserves no less attention.

This study was funded by the Department of Health's National Institute of Health Research (NIHR) as part of its programme grants for applied research funding scheme. We thank members of the NIHR programme grant team: Chantal Orgeas, Elaine Boyle, and Julie Faulkes (University of Leicester) for helpful advice and comments throughout the study. We also thank Shona Golightly (Centre for Maternal and Child Enquiries) for help with information on neonatal and infant deaths and comments on earlier drafts of the paper. 
Contributors: LKS did the statistical analysis and wrote the first draft of the paper. LKS, BNM, ESD, and DJF made substantial contributions to conception and design. LKS, ESD, and AS were responsible for acquisition of data. BNM made a substantial contribution to analysis. LKS, BNM, ESD, and DJF contributed to interpretation of data. All authors contributed to drafting the article or revising it critically for important intellectual content and approved the final manuscript. LS is the guarantor.

Funding: UK Department of Health's National Institute of Health Research. Competing interests: All authors have completed the Unified Competing Interest form at www.icmje.org/coi_disclosure.pdf (available on request from the corresponding author) and declare: all authors had financial support from UK Department of Health's National Institute of Health Research for the submitted work; no financial relationships with any organisations that might have an interest in the submitted work in the previous 3 years; no other relationships or activities that could appear to have influenced the submitted work.

Ethical approval: Not needed.

Data sharing: No additional data available.

1 Judge K. Inequalities in infant mortality: patterns, trends, policy responses and emerging issues in Canada, Chile, Sweden and the United Kingdom. Health Sociol Rev 2009;18:12-24.

2 DeFranco EA, Lian M, Muglia LA, Schootman M. Area-level poverty and preterm birth risk: a population-based multilevel analysis. $B M C$ Public Health 2008;8:316.

3 Department of Health. Tackling health inequalities: a programme for action. Stationery Office, 2003.

4 Department of Health. Review of the health inequalities infant mortality PSA target. Department of Health, 2007.

5 Guildea ZE, Fone DL, Dunstan FD, Sibert JR, Cartlidge PH. Social deprivation and the causes of stillbirth and infant mortality. Arch Dis Child 2001;84:307-10.

6 Oakley L, Maconochie N, Doyle P, Dattani N, Moser K. Multivariate analysis of infant death in England and Wales in 2005-06, with focus on socio-economic status and deprivation. Health Stat $Q$ 2009;42:22-39.

7 Moser K. Gestation-specific infant mortality by social and biological factors among babies born in England and Wales in 2006. Health Stat Q 2009;42:78-87.

8 Smith LK, Draper ES, Manktelow BN, Dorling JS, Field DJ. Socioeconomic inequalities in very preterm birth rates. Arch Dis Child Fetal Neonatal Ed 2007;92:F11-4.

9 March of Dimes Foundation. White paper on preterm birth: the global and regional toll. March of Dimes Foundation, 2009.

10 Janghorbani M, Stenhouse E, Millward A, Jones RB. Neighborhood deprivation and preterm birth in Plymouth, UK. J Matern Fetal Neonatal Med 2006;19:85-91.

11 Keeling JW, MacGillivray I, Golding J, Wigglesworth J, Berry J, Dunn PM. Classification of perinatal death. Arch Dis Child 1989;64:1345-51
12 Noble M, Wright G, Dibben C, Smith G, McLennan D, Anttila C, et al. Indices of deprivation 2004: report to the office of the deputy prime minister. Neighbourhood Renewal Unit, 2004.

13 Lumley T, Kronmal R, Ma S. Relative risk regression in medical research: models, contrasts, estimators, and algorithms. University of Washington, 2006. (UW Biostatistics Working Paper Series.)

14 Shaw M, Galbardes B, Lawlor D, Lynch J, Wheeler B, Davey Smith G. The handbook of inequality and socioeconomic position. Policy Press, 2007.

15 Oehlert GW. A note on the delta method. Am Stat 1992;46:27-9.

16 Field DJ, Dorling JS, Manktelow BN, Draper ES. Survival of extremely premature babies in a geographically defined population: prospective cohort study of 1994-9 compared with 2000-5. BMJ 2008;336:1221-3

17 Tommiska V, Heinonen K, Lehtonen L, Renlund M, Saarela T, Tammela O, et al. No improvement in outcome of nationwid extremely low birth weight infant populations between 1996-1997 and 1999-2000. Pediatrics 2007;119:29-36.

18 Vanhaesebrouck P, Allegaert K, Bottu J, Debauche C, Devlieger H, Docx M, et al. The EPIBEL study: outcomes to discharge from hospital for extremely preterm infants in Belgium. Pediatrics 2004;114:663-75.

19 Smith LK, Draper ES, Manktelow BN, Field DJ. Deprivation and infection among spontaneous very preterm births. Obstet Gynecol 2007;110:325-9.

20 Nuffield Council on Bioethics. Critical care decisions in fetal and neonatal medicine: ethical issues. Nuffield Council on Bioethics, 2006.

21 Rankin J, Pattenden S, Abramsky L, Boyd P, Jordan H, Stone D, et al. Prevalence of congenital anomalies in five British regions, 1991-99. Arch Dis Child Fetal Neonatal Ed 2005;90:F374-9.

22 Neasham D, Dolk H, Vrijheid M, Jensen T, Best N. Stillbirth and neonatal mortality due to congenital anomalies: temporal trends and variation by small area deprivation scores in England and Wales, 1986-96. Paediatr Perinat Epidemiol 2001;15:364-73.

23 Vrijheid M, Dolk H, Stone D, Abramsky L, Alberman E, Scott JE. Socioeconomic inequalities in risk of congenital anomaly. Arch Dis Child 2000;82:349-52.

24 Guildea ZE, Fone DL, Dunstan FD, Sibert JR, Cartlidge PH. Social deprivation and the causes of stillbirth and infant mortality. Arch Dis Child 2001;84:307-10.

25 Gray R, Bonellie SR, Chalmers J, Greer I, Jarvis S, Kurinczuk J), et al. Contribution of smoking during pregnancy to inequalities in stillbirth and infant death in Scotland 1994-2003: retrospective population based study using hospital maternity records. BMJ 2009;339:b3754.

26 Field D, Draper ES, Fenton A, Papiernik E, Zeitlin J, Blondel B, et al. Rates of very preterm birth in Europe and neonatal mortality rates. Arch Dis Child Fetal Neonatal Ed 2009;94:F253-6.

27 Muglia LJ, Katz M. The enigma of spontaneous preterm birth. N Engl J Med 2010;362:484, 529-35.

28 lams JD, Romero R, Culhane JF, Goldenberg RL. Primary, secondary, and tertiary interventions to reduce the morbidity and mortality of preterm birth. Lancet 2008;371:164-75. 